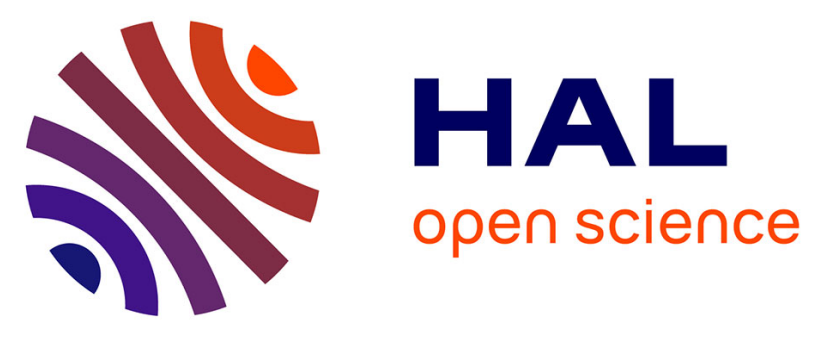

\title{
Deviation from threshold model in ultrafast laser ablation of graphene at sub-micron scale
} Abel Villalba, Chen Xie, Roland Salut, Luca Furfaro, Remo Giust, M. Jacquot, P.A Lacourt, John Michaël Dudley, François Courvoisier

\section{To cite this version:}

Abel Villalba, Chen Xie, Roland Salut, Luca Furfaro, Remo Giust, et al.. Deviation from threshold model in ultrafast laser ablation of graphene at sub-micron scale. Conference on Lasers and ElectroOptics and European Quantum Electronics Conference, Jun 2015, Munich, Germany. hal-02134929

\author{
HAL Id: hal-02134929 \\ https://hal.science/hal-02134929
}

Submitted on 20 May 2019

HAL is a multi-disciplinary open access archive for the deposit and dissemination of scientific research documents, whether they are published or not. The documents may come from teaching and research institutions in France or abroad, or from public or private research centers.
L'archive ouverte pluridisciplinaire $\mathbf{H A L}$, est destinée au dépôt et à la diffusion de documents scientifiques de niveau recherche, publiés ou non, émanant des établissements d'enseignement et de recherche français ou étrangers, des laboratoires publics ou privés. 


\title{
Deviation from threshold model in ultrafast laser ablation of graphene at sub-micron scale
}

\author{
A. Gil-Villalba, C. Xie, R. Salut, L. Furfaro, R. Giust, M. Jacquot, P.A. Lacourt, \\ J.M. Dudley, and F. Courvoisier a)
}

Institut FEMTO-ST, UMR 6174 CNRS Universite de Bourgogne Franche-Comte, 25030 Besançon Cedex, France

\begin{abstract}
We investigate a method to measure ultrafast laser ablation threshold with respect to spot size. We use structured complex beams to generate a pattern of craters in CVD graphene with a single laser pulse. A direct comparison between beam profile and SEM characterization allows us to determine the dependence of ablation probability on spot-size, for crater diameters ranging between $700 \mathrm{~nm}$ and $2.5 \mu \mathrm{m}$. We report a drastic decrease of ablation probability when the crater diameter is below 1 $\mu \mathrm{m}$ which we explain by free-carrier diffusion.
\end{abstract}

Graphene nanopatterning has a very wide range of applications for next generation technology: photonics, displays or solar energy ${ }^{1}$. Mass fabrication of such devices requires low cost and fast processes operating at micro- to nano-metric scales. Existing methods of patterning graphene include focused ion beam (FIB), ${ }^{2}$ Helium ion lithography (HIL), ${ }^{3,4}$ electron beam lithography in conjunction with reactive ion etching, ${ }^{5} \mathrm{UV}$ nanoimprint lithography ${ }^{6}$ and direct etching with an electron beam in a transmission electron microscope (TEM). ${ }^{7}$ These methods are suitable for patterning in the tens of nanometer range. Most of these procedures require vacuum and involve multiple steps. Laser processing is extremely attractive especially because it does not require vacuum nor harmful chemicals. It is fast, single step, contact-free and easily reconfigurable. Ultrashort laser pulses have already been identified as promising ways for processing thin films for craters diameters down to $100 \mathrm{~nm} .{ }^{8,9}$ In addition, massive parallelization is now possible with multiple beam interference lithography. ${ }^{10-14}$

Ultrashort laser patterning of graphene has been already investigated in single shot for diameters on the order of $1-2 \mu \mathrm{m}$ and channels in the order of $0.5-1 \mu \mathrm{m}$ width in damage accumulation. ${ }^{15,16}$ Nanopatterning with exceptional resolution in the sub 100-nm range has been demonstrated in the thermal accumulation regime. ${ }^{17}$ Fabrication of nano-ribbons in the range of tens of nanometers in width have been demonstrated with this latter approach. The processing speed is however incompatible with industrial scale fabrication. At present, single shot fast patterning of graphene at sub-micrometer scale is very attractive but has not been investigated. A number of applications in photonics and optoelectronics would require arrays of holes ranging from $100 \mathrm{~nm}$ to $1 \mu \mathrm{m} .^{18,19}$

a) Author to whom correspondence should be addressed. Electronic mail: francois.courvoisier@ @emto-st.fr 
The technological importance of graphene partly comes from the high intrinsic electron mobility and heat diffusion coefficients. ${ }^{20,21}$ However, when energy is deposited in the material by a laser pulse, the high diffusion coefficients rapidly spread the deposited energy. This is potentially major drawback against obtaining high confinement of laser energy deposition that is necessary for extremely localized ablation. It is therefore important to investigate ultrashort pulse ablation in single shot regime at sub-micrometer scale.

Several methods have been developed to investigate the ablation threshold. Regression of crater diameters with fluence averages the fluence threshold over several holes. ${ }^{22}$ Recently, the ablation threshold has been retrieved from the comparison between the beam profile and the damage observed in situ by optical microscopy. ${ }^{23}$ In back-geometry irradiation it has been demonstrated reproducible submicron ablation controlling the crater diameter as a function of the focal plane relative to the surface. ${ }^{9}$ This approach is however inapplicable for craters with sub- $\mu \mathrm{m}$ dimensions and the relative magnification between beam and damage is a potential source of flaw for circularly symmetric damages.

In this Letter, we report a method based on single shot illumination of the sample with a well-characterized complex beam pattern and imaging of the damages by Scanning Electron Microscopy (SEM). The beam pattern is obtained by multiple interference, which define $\sim 40$ intensity spots with different sizes and peak intensities. These produce in single shot a set of craters with diameters ranging between 0.7 to $2.5 \mu \mathrm{m}$. This approach allows us to directly explore with better confidence the relative ablation threshold and probability of ablation depending on the size, without the ambiguity on pulse energy fluctuations or sample-to-focus critical positioning issue. ${ }^{9}$ We use an interference pattern which defines lobes that are almost non-diffracting, which also removes the latter constraint on positioning. ${ }^{24}$ In addition, during the fitting procedure, the ambiguity on the relative magnification between beam and sample images is dropped out since the distance between the craters is fixed by the distance between the intensity spots.

With this approach, we have determined the ablation threshold of CVD graphene under ultrafast laser pulse irradiation and investigated the evolution of the ablation probability versus the size of the damage. We show that below crater dimensions of $\sim 1 \mu \mathrm{m}, \mathrm{CVD}$ graphene exhibits a strong deviation from the intensity-threshold model. The ablation probability drops, which is interpreted in terms of both electron-hole diffusion and ultrafast scattering with phonons. ${ }^{25}$

Our experimental setup is represented in Fig. 1. We use a Ti:Sa regenerative amplifier laser system delivering $130 \mathrm{fs}$ pulses at $800 \mathrm{~nm}$. A pulse picker allows for operating in single-shot mode. The pulse to pulse energy variation was independently characterized at less than $2 \%$. Spatial beam shaping is realized with an optically addressed Spatial Light Modulator (SLM, Hamamatsu PAL-SLM) associated to a demagnification optical arrangement (factor 1/278). The setup also includes spatial Fourier filtering and the beam is characterized by using a second microscope objective as in Reference 26. The absolute 
intensity distribution is determined by calibrating the response of the high-dynamical range (16 bits) CCD camera. The pulse duration is calibrated by a two-photon absorption photodiode at the sample site and monitored by chirping the pulse with the compressor of the laser chain.

We choose a 3 -fold symmetry interference beam pattern. It is generated from a 3 -facet pyramid phase mask ${ }^{10}$ as shown in Fig. 2(a). Fig. 2(b) shows the intensity distribution at the sample plane, where the interference field is the widest. The interfering beamlets propagate at an angle of $9^{\circ}$ with respect to the optical axis. The 3 -wave interference generates lobes with sinusoidal profile, with a size quasi-constant from spot to spot. The inhomogeneity between the lobe intensities comes from the limited size of the beam illuminating the Spatial Light Modulator. The sample is positioned in the interference region with an absolute precision of less than $2 \mu \mathrm{m}$ with respect to the beam position. ${ }^{24}$ At this position, the intensity distribution varies by less than $6 \%$ over the range of positioning error. The sample is commercial CVD graphene monolayer on a glass substrate. In our experiments, the fluence is kept much below the ablation threshold of the substrate.

We performed 3 sets of ablations at pulse durations of $130 \mathrm{fs}, 1 \mathrm{ps}$ and $3 \mathrm{ps}$ where we varied the pulse energy and repeated the experiment 5 times in identical conditions. The sample was characterized by SEM, as shown in Fig. 3.

The ablation fluence threshold of CVD graphene was determined by comparing the beam fluence distribution and the SEM image of the ablated sample. We note the images of CVD graphene have a high dynamical range so that it is possible to discriminate between ablated areas, laser damaged areas and un-modified areas. ${ }^{27}$

In Figure 3, we show as an example the image of the ablated sample and beam fluence distribution for one of our data points. After scaling and alignment of SEM and beam images, we implemented two different numerical methods to determine the fluence threshold for ablation. In the first method, we numerically evaluated the correspondence between ablated sample areas and beyond-threshold beam areas. Identically, we evaluated the correspondence between non-ablated areas and below-threshold beam areas. The correlation is calculated as the normalized sum of the logical equalities (XNOR function) pixel per pixel over the whole image area. The matching percentage varying as a function of the fluence threshold value is shown for one pulse energy in figure 4(a). Since the image totals a large area where no ablation occurs where the beam fluence is negligible, the correlation percentage is naturally high $(98 \%)$. The maximum of the correlation curve is still very well defined and provides the value of the threshold. Fig. 4(b) shows the residues of the correspondence at the ablation threshold: the green part shows the matching areas, red areas are the points where ablation is expected from the threshold and do not occur. Symmetrically, blue areas are the points where ablation is not expected but did occur. Since we focus on ablation, the areas where graphene was damaged but not removed was counted as unmodified in this procedure. We note that 
within the range of experimental conditions reported here, we never observed graphene folding, whereas it was observed for different beam shapes ${ }^{27,28}$. A possible origin of this difference is the influence of laser deposited energy profile.

We readily observe that for the largest diameters in the central part of the beam, the residues reveal the absence of systematic errors since blue/red areas are almost randomly distributed for damage diameters larger than $1 \mu \mathrm{m}$. In Fig. 5 we report in red the values of the ablation threshold obtained with the procedure described above and that was averaged over 25 images for each pulse duration (i.e. 5 different input pulse energies between 1.00 and $2.00 \mu \mathrm{J}$, repeated 5 times). This effectively provides an average over $\sim 1250$ craters. The error bar takes into account the deviation between the images, the errors originating from positioning and energy characterization, and the resolution of the peak of the correlation curve. This measurement has been repeated for different pulse durations (130 fs, 1 ps, 3ps) and we obtain the ablation fluence threshold of $139 \pm 7 \mathrm{~mJ} / \mathrm{cm}^{2}$ for $130 \mathrm{fs}$ pulse duration, $166 \pm 9 \mathrm{~mJ} / \mathrm{cm}^{2}$ for $1 \mathrm{ps}$ and $190 \pm 9 \mathrm{~mJ} / \mathrm{cm}^{2}$ for 3 ps. These results are in good agreement with previous results, ${ }^{15,27,28}$ ranging between $100-210 \mathrm{~mJ} / \mathrm{cm}^{2}$, reported on single-shot ablation threshold of CVD graphene samples for similar pulse durations but on wider diameters. A similar procedure has been used to characterize multishot processing of stripes in graphene ${ }^{29}$.

Importantly, our technique allows for investigating the influence between spots. When the input pulse energy is increased, the areas where the fluence threshold is reached get larger and larger while the distance between neighboring areas obviously reduces. Within our experimental data, the boundaries between independent spots varied from 0.8 to $2.5 \mu \mathrm{m}$. Within this range, the change in ablation threshold fluence was not significant and remained below the error bar.

For the second method, where the results are shown in blue in Fig. 5, we numerically determined the positions of the contour boundaries between ablated and non-ablated areas on SEM images. The fluence threshold was measured as the average of the fluence value over the contours (again over $\sim 1250$ craters). The results shown in blue in Fig. 5 are in good agreement with the previous one.

From a closer look at Fig. 4(b), we observe that there is a quantitative difference for the matching between the craters with the largest diameters, located in the central part of the pattern and the craters with the smallest diameters, located in the outer part of the pattern. Indeed, while the firsts show a mismatch of typically less than $15 \%$ of the diameter $(0.15$ to $0.65 \mu \mathrm{m}$ mismatch for crater diameter ranging between 1.4 and $2.6 \mu \mathrm{m})$, the second have a discrepancy reaching typically $50 \%(0.25$ to $0.65 \mu \mathrm{m}$ mismatch for crater diameter ranging between 0.7 and $0.9 \mu \mathrm{m}$ ). Several entirely red disks of $\sim 400-700 \mathrm{~nm}$ in diameter are visible, where ablation is expected from the ablation threshold but did not occur.

To quantitatively characterize this behavior, we tracked the same intensity lobes in the beam over 5 sample processing experiments with identical parameters. For each lobe, we can determine the ablation probability. The reference parameter was 
taken as the mean diameter of the lobe over the fluence threshold. This procedure provides, for each pulse energy, a set of $\sim$ 40 values of ablation probability with their respective diameters. In Fig. 6, we plot as a histogram the evolution of the ablation probability as a function of diameter for input pulse energies from $1 \mu \mathrm{J}$ to $2 \mu \mathrm{J}$ and duration 130 fs, 1 ps and 3 ps. The histogram therefore compiles results where the peak fluence values within the laser spots, range from $150 \mathrm{~mJ}^{\mathrm{c}} \mathrm{cm}^{-2}$ to $320 \mathrm{~mJ} . \mathrm{cm}^{-2}$. We observe that below a diameter of $\sim 1 \mu \mathrm{m}$, the ablation probability strongly decreases. This behavior is similar for all pulse durations. We note that the structuration of the beam has negligible influence on the variation of the pulse duration over the different lobes. ${ }^{30}$

A variation of the ablation probability of bulk fused silica has been reported in other conditions with varying numerical aperture. ${ }^{31}$ The decrease of the probability with spot-size decrease was attributed to the reduction of the probability of the ablation volume to meet a structural defect. ${ }^{32}$ In our case, the equivalent NA is constant at 0.16 , but the difference in amplitude from spot to spot provides an equivalent difference in size of the over-threshold area. Spots with over-threshold diameter are obviously more prone to meet defects.

In addition, we highlight the role of free-electron diffusion. The typical diffusion coefficient for the $2 \mathrm{D}$ free-electron gas at $\sim 1 \mathrm{eV}$ is $\mathrm{D} \sim 5500 \mathrm{~cm}^{2} / \mathrm{s}^{33,34}$ Within $100 \mathrm{fs}$, a gaussian distribution of free-electrons with initial diameter $400 \mathrm{~nm}$ at $1 / \mathrm{e}$ expands to $1 \mu \mathrm{m}$ diameter while reducing the peak density by a factor of 6.5 . In contrast, if the initial distribution is $2 \mu \mathrm{m}$ in diameter, it expands only to $2.2 \mu \mathrm{m}$ in the same time and the peak electron density drops only by a factor of 1.2 , i.e. 5 times less than in the previous case. This comparison shows that the high free-carrier diffusion can explain the difference in ablation probability at diameters below $1 \mu \mathrm{m}$. Free-electron distributions with a small diameter are highly sensitive to local variations of the diffusion coefficient. In our interpretation, structural defects locally reduce the free-electron mobility, and effectively increase the ablation probability. ${ }^{35}$ The influence of inhomogeneities on multi-shot laser processing has also been raised in reference 29. In our view, the discrepancies (blue, red areas) observed in the correlation map of figure 4(b) can be interpreted as originating from presence/absence of localized defects. Additional diffusion mechanisms such as transfer to phonons might also influence the overall ablation process. More investigations are required to clarify then origin of the ablation probability drop.

In conclusion, we have developed a method with two different approaches to investigate the variation of ablation probability with spot size operable at sub- $\mu \mathrm{m}$ scale. Our results for CVD graphene show a drastic decrease of the ablation probability below $1 \mu \mathrm{m}$ for the three pulse durations investigated, ranging between 130 fs and 3 ps. This suggests the underlying mechanism is faster than 130 fs. We showed our results can be explained by the high carrier diffusion coefficient 
in graphene. We anticipate this technique and our results will impact on laser surface nanopatterning of thin films and graphene.

We acknowledge fruitful discussions with Prof. Michel Devel. The authors acknowledge funding from Region FrancheComte, French ANR, contract 2011-BS04-010-01 NANOFLAM. This work has been performed in cooperation with the Labex ACTION program (contract ANR-11-LABX-01-01). This work was partly supported by the French RENATECH network.

\section{References}

${ }^{1}$ F. Bonaccorso, Z. Sun, T. Hasan, and A.C. Ferrari, Nature Photon. 4, 611 (2010).

${ }^{2}$ A. Hemanouche, A. Morin, E. Bouhis, B. Toury, E. Tarnaud, J. Mathé, P. Guégan, A. Madouri, X. Lafosse, C. Ulysse, et al, Microelectronic Engineering 121, 87 (2014)

${ }^{3}$ D.C. Bell, M.C. Lemme, L. A. Stern, J.R. Williams, and C. M. Marcus, Nanotech. 20, 455301 (2009)

${ }^{4}$ Y. M. Han, B. Özyilmaz, Y. Zhang, and Philip Kim, Phys. Rev. Lett. 98, 206805 (2007)

${ }^{5}$ L. A. Ponomarenko, F. Schedin, M. I. Katsnelson, R. Yang, E. W. Hill, K. S. Novoselov, and A. K. Geim, Science 320, 356 (2008).

${ }^{6}$ P. Kumar, K. S. Subrahmayam, and C. N. R. Rao, Mat. Expr. 1,3(2011)

${ }^{7}$ C. Wang, K. J. Morton, Z. Fu, W.-D. Li, and S. Y. Chou, Nanotech. 22 (2011) 445301

${ }^{8}$ A. P. Joglekar, H. Liu, E. Meyhöfer, G. Mourou, and A. J. Hunt, Proc. Natl. Acad. Sci; U.S.A. 101, 5856 (2004)

${ }^{9}$ L. Mercadier, D. M. Rayner, and P. B. Corkum, Phys. Rev. Appl. 2, 034001 (2014)

${ }^{10}$ J. de Boor, N. Geyer, U. Gösele, and V. Schmidt, Opt. Lett. 34, (2009)

${ }^{11}$ C. Lu, and R. H. Lipson, Laser \& Phot. Rev. 4, 568 (2010)

${ }^{12}$ Y. Nakata, K. Murakawa, K. Sonoda, K. Momoo, and N. Miyanaga, Appl. Phys. A 112, 191 (2013)

${ }^{13}$ Q. Zhou, W. Yang, F. He, R. Stoian, R. Hui, and G. Cheng., Opt. Exp. 21, 183641 (2013)

${ }^{14}$ S. Hasegawa, Y. Hayasaki, International Journal of Optomechatronics 8, 73 (2014)

${ }^{15}$ A. Roberts, D. Cormode, C. Reynolds, T. Newhouse-Illige, B.J. LeRoy, and A. Sandhu, App. Phys. Lett. 99, 051912 (2011)

${ }^{16}$ R. Sahin, E. Simsek, and S. Akturk, Appl. Phys. Lett. 104, 053118 (2014)

${ }^{17}$ R. J. Stohr, R. Kolesov, K. Xia, and J. Wrachtrup, ACS Nano 5, 5141 (2011).

${ }^{18}$ W. Wang, T. Christensen, A.-K. Jauho, K. S. Thygesen, M. Wubs, and N. A. Mortensen, Sci. Rep. 5, 9535 (2015)

${ }^{19}$ A. Y. Nikitin, F. Guinea, and L. Martin-Moreno, Appl. Phys. Lett. 101, 151119 (2012) 
${ }^{20}$ S. V. Morozov, K. S. Novoselov, M. I. Katnelson, F. Schedin, D.C. Elias, J.A. Jaszczak, and A. K. Geim, Phys. Rev. Lett. 100, 016602 (2008)

${ }^{21}$ A. A. Balandin, S. Ghosh, W. Bao, I. Calizo, D. Teweldebrhan, F. Miao, and C. N. Lau, Nano Lett. 8, 902 (2008)

${ }^{22}$ N. Sanner, O. Utéza, B. Bussiere, G. Coustillier, A. Leray, T. Itina, M. Sentis, Appl. Phys. A 94, 889 (2009)

${ }^{23}$ B. Mangote, L. Gallais, M. Zerrad, F. Lemarchand, L. H. Gao, M. Commadré, and M. Lequime, Rev. Scient. Instr. 83, 013109 (2012)

${ }^{24}$ F. Courvoisier, P.-A. Lacourt, M. Jacquot, M. K. Bhuyan, L. Furfaro, and J. M. Dudley, Opt. Lett. 34, 3163 (2009)

${ }^{25}$ M. Breusing, C. Ropers, and T. Elsaesser, Phys. Rev. Lett. 102, 086809 (2009)

${ }^{26}$ C. Xie, V. Jukna, C. Milian, R. Giust, I. Ouadghiri-Idrissi, T. Itina, J. M. Dudley, A. Couairon, and F. Courvoisier, Sci. Rep. 5, 8914 (2015)

${ }^{27}$ B. Wetzel, C. Xie, P.-A. Lacourt, J. M. Dudley, and F. Courvoisier, App. Phys. Let. 103, 241111 (2013).

${ }^{28}$ J-H. Yoo, J.B. Park, H. Jeon, and C.P. Grigoropoulos, App. Phys. Lett. 100, 233124 (2012).

${ }^{29}$ R. Sahin, S. Akturk, E. Simsek, Appl. Phys. A 116, 555 (2014)

${ }^{30}$ L. Froehly, M. Jacquot, P.A. Lacourt, J.M. Dudley, F. Courvoisier, J. Opt. Soc. Am. A 31,790 (2014)

${ }^{31}$ J. B. Ashcom, R. R. Gattass, C. B. Schaffer, and E. Mazur; J. Opt. Soc; Am. B 23, 2317 (2006)

${ }^{32}$ S. Martin, A. Hertwig, M. Lenzner, J. Krüger, abd W. Kautek, Appl. Phys. A 77, 883 (2003)

${ }^{33}$ B. A. Ruzicka, S. Wang, J. Liu, K.-P. Loh, J. Z. Wu, and H. Zhao, Opt. Mat. Express 2, 708 (2012)

${ }^{34}$ B. A. Ruzicka, S. Wang, L. K. Werake, B. Weintrub, K. P. Loh, and H. Zhao, Phys. Rev. B 82, 195414 (2010)

${ }^{35}$ J. Noack, and A. Vogel, J. Quant. Elect. 35, 1156 (1999) 


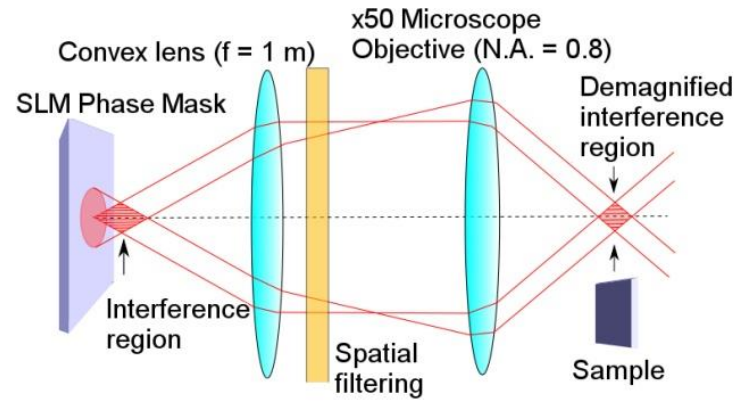

FIG. 1. Experimental setup. The lens and microscope objective form a $4 \mathrm{f}$ telescope that demagnifies the beam and increases the waves crossing angle. Spatial filtering allows for eliminating unmodulated zeroth-order from the Spatial Light Modulator (SLM).

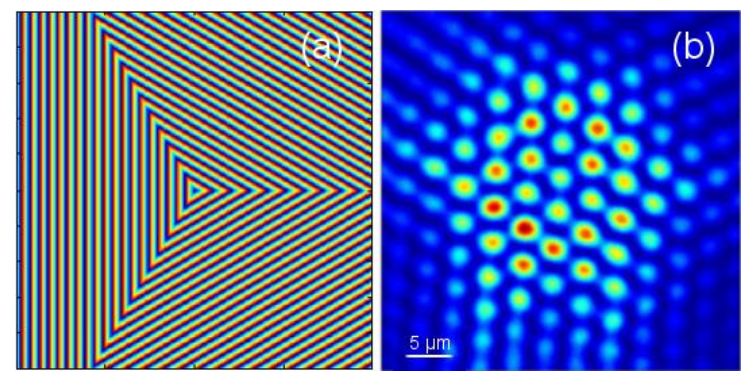

FIG.2. (a) Phase mask loaded at the SLM to generate the three-beam interference pattern and (b) scanned intensity beam profile recorded on the CCD camera.

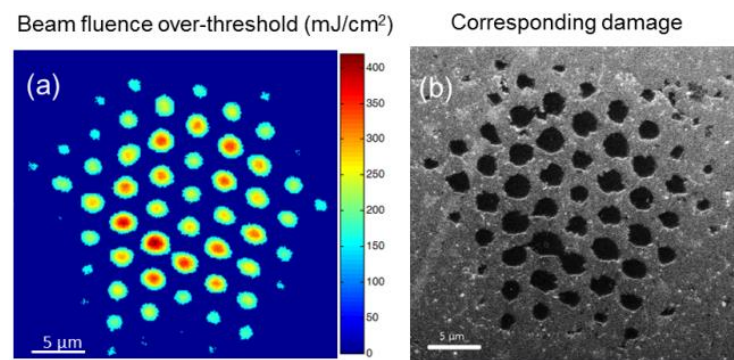

FIG. 3. Comparison of fluence distribution over threshold (a) with the SEM image of the damage (b) for a given intensity distribution, pulse duration (130 fs) and input pulse energy $(1.3 \mu \mathrm{J})$. 

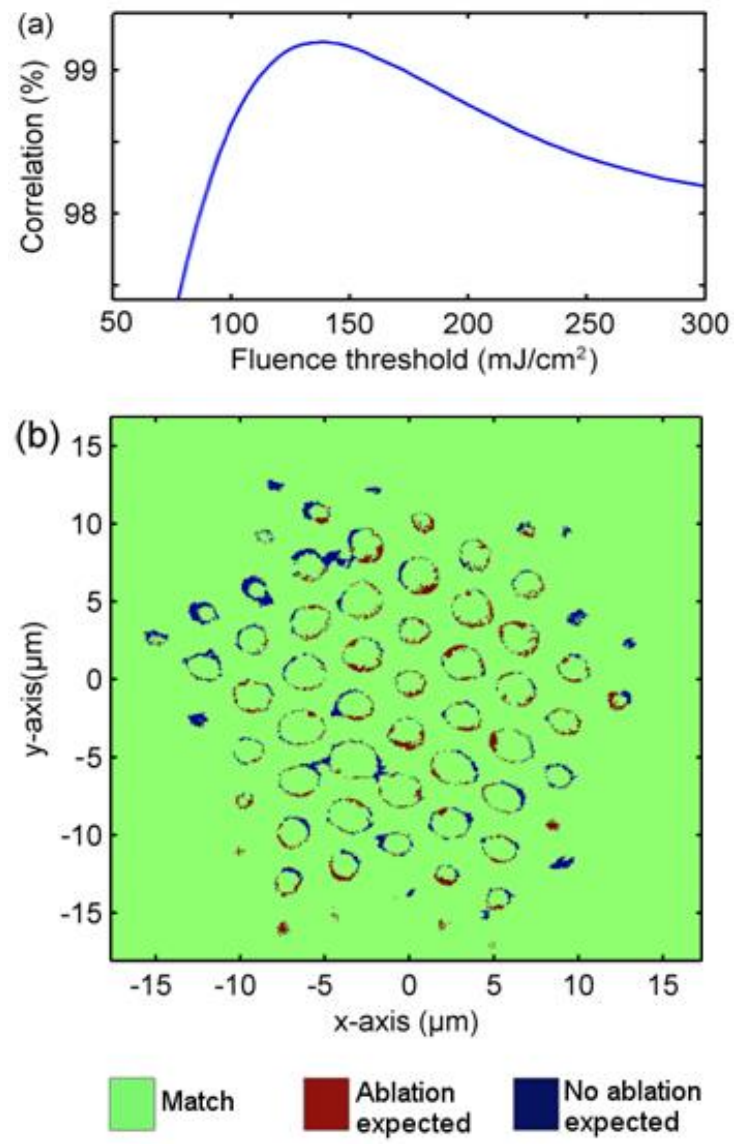

FIG.4. (a) Correlation between the ablated sample image and the scanned beam image applying different fluence thresholds, for a given pulse duration (130 fs) and input pulse energy $(1.3 \mu \mathrm{J})$. (b) Image of the spatial distribution of the residues: green color for matching areas, red (resp. blue) for areas where the fluence is higher (resp. lower) than the threshold but ablation does not occur (resp. did occur). This image corresponds to the peak of the correlation in (a), for a fluence threshold of $(137 \pm 9) \mathrm{mJ} / \mathrm{cm}^{2}$.

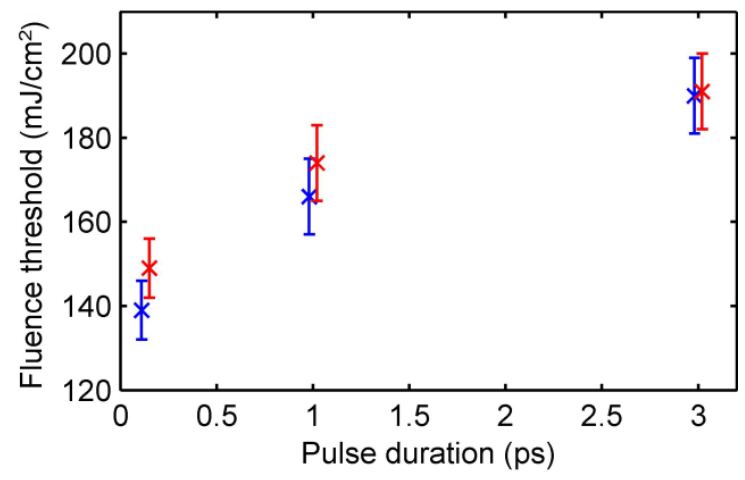

FIG 5. Comparison between the ablation threshold in monolayer graphene for different pulse duration determined by the best matching over different fluence thresholds technique (red) and by the mean fluence at the ablated contour (blue). 


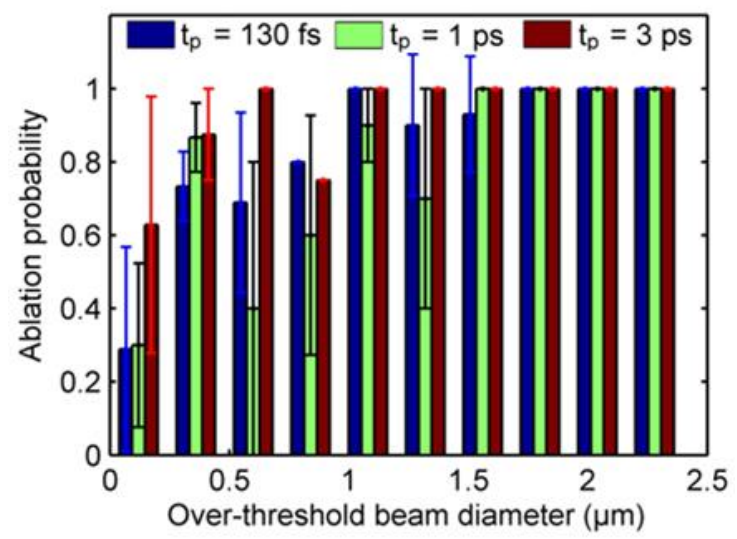

FIG. 6. Probability of ablation in a disk as a function of the beam diameter above the fluence threshold for a pulse duration of $130 \mathrm{fs}$ (blue), 1 ps (green) and 3 ps (red). Error bars show one standard deviation. For the largest diameters, the ablation probability is actually $100 \%$ of our tests, resulting in zero-width error bar. 